\title{
Flip Chip Technologies and Their Applications in MEMS Packaging
}

\author{
Hai-ying Wang, Yi-long Bai \\ LNM, Institute of Mechanics, Chinese Academy of Sciences, Beijing 100080, China, \\ Email:why@Inm.imech.ac.cn
}

\begin{abstract}
The high cost and undesirable bulky packages have been and continues to be major stumbling blocks in commercializing MEMS products. Flip chip technologies are very promising techniques for MEMS packaging. This paper presents a brief introduction of flip chip technologies as well as examples of successful applications of flip chip technologies in MEMS (e.g. RF and optical MEMS, micro sensors) packaging. The result shows that, although MEMS packaging is much more complicated than that for microelectronics, flip chip technologies can be applicable means of integrating MEMS devices. It is also shown that to meet different packaging requirements of various MEMS devices, bumping materials, bumping process and joining methods must be chosen carefully. Issues, such as package deformation, temperature distributions, heat sink placement, are all very crucial for the performance of MEMS devices and should be considered in MEMS packaging design.
\end{abstract}

Keywords: MEMS, packaging, flip chip technologies

\section{Introduction}

The advance of microelectromechanical system (MEMS) in the past decade has been truly impressive in both its pace and the variety of new applications. Many MEMS devices must communicate with the outside world by methods like electronic and optical signals. MEMS packaging provides the interface between components and the overall system. In addition, most MEMS devices are minute and highly complex, involving structural components in the form of delicate thin beams and diaphragms. Excessive stresses and deformation of these components induced by the operating loads can seriously affect the performance of these devices. Proper designed packaging of MEMS devices therefore will not only ensure the expected performance of the products, but also make them reliable and competitive in the marketplace.
Packaging is one of the most critical factors in commercializing MEMS products [1]. For example, the cost of packaging a micro pressure sensor can vary from $20 \%$ to as high as $95 \%$ of the product's total cost. Often, the physical size of a micromachined sensor is dwarfed by the size of its final package. A sensing or actuating element of the size of a few microns can end up in a package with an overall size in centimeters. Consequently, most of the benefits of miniaturization are diminished due to inadequate packaging. The high cost and the undesirable bulky packages have been major stumbling blocks in commercializing MEMS products.

The lack of MEMS packaging standards and design methodology is the principal reason for the inadequate packaging for MEMS devices. Worse yet, MEMS devices are so applicationspecific that hard-earned solutions for one device don't necessarily translate to other 
products. These facts make MEMS packaging the greatest challenge faced by their researchers. In recent years, researchers have developed some novel packaging technologies for various MEMS devices [2,3]. Among them, flip chip technologies is one of the promising techniques for MEMS packaging.

\section{Flip chip technologies}

Flip chip technologies were introduced by IBM in 1960s, and are well established in microelectronic packaging now. Flip chip microelectronic assembly is the direct electrical connection of face-down (hence, "flipped") electronic components onto substrates, circuit boards, or carriers, by means of conductive bumps on the chip bond pads or conductive epoxy filling in the gap between the chip and substrate. Flip chip technologies can achieve packages with small size, high performance, great I/O flexibility, low cost, and high reliability. These significant advantages make flip chip packaging very popular in microelectronic field [4].

A variety of flip chip technologies are available today. These technologies vary in the packaging structure, bump material, as well as manufacturing process. The variety enables flip chip technologies very flexible for different applications, including the packaging of MEMS devices.

\section{Applications in MEMS packaging}

\section{1 micromachined conductive polymer bumps}

Because of their inherent advantages, flip chip technologies are preferred for the mounting or inverting of photonic devices on optical communication systems, where precise input/output coupling is required between photonic devices and optical waveguide or optic fibers (Fig. 1). However, either the conventional solder bump or the screen printing-based polymer bump flip chip technology has drawbacks like high soldering temperature, complicated processing steps, or crude bumping alignment. Therefore, Kwang et al. developed a conductive polymer flip chip technique to address possible problems caused by the solder bump technique and the screen printing-based

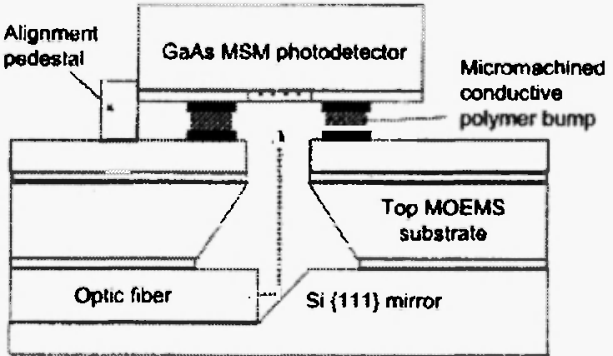

Fig. I Schematic diagram of an optical I/O coupler by use of flip chip bonding technique

polymer bonding technique [5].

Fig.2 illustrates fabrication steps of the micromachined bumps employing thick photoresist bump-holes as molding patterns. Initially $\mathrm{Cr} / \mathrm{Au}$ contact metal pad lines and pads for the conductive polymer bumps were formed by lift-off on a silicon dioxide layer, which serve as an insulation layer. Following this, the thick photoresist was patterned for bumpholes. After the lithography, thermoplasitc

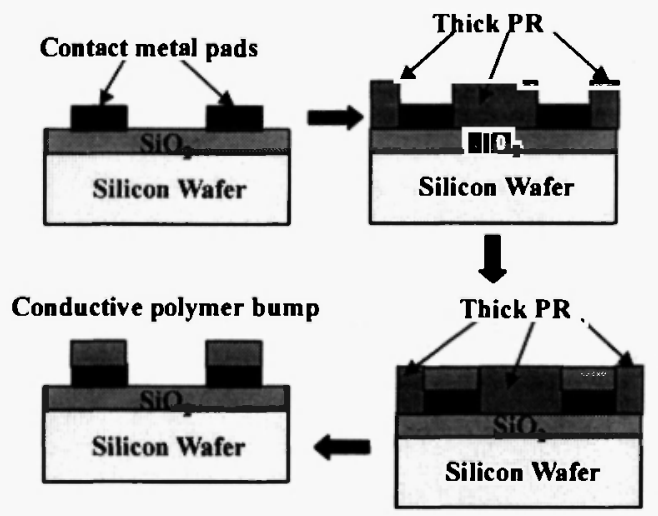

Fig.2 Fabrication steps of conductive polymer bumps conductive polymer materials were applied into the bump-hole patterns. Then, the wafer was cured at $100^{\circ} \mathrm{C}$ for about $15 \mathrm{~min}$. Due to the different curing conditions between the thick photoresists and the conductive polymers, the photoresist molds can be removed, leaving the conductive polymer bumps on the contact metal pads. After this selective curing, photoresist molds were stripped away in conventional 
phtoresist stripper, leaving the polymer bumps on the contact metal pads. After that, the wafer was fully cured to achieve a better conductivity for the conductive polymers. The wafer was now ready to go for dicing and flip chip bonding.

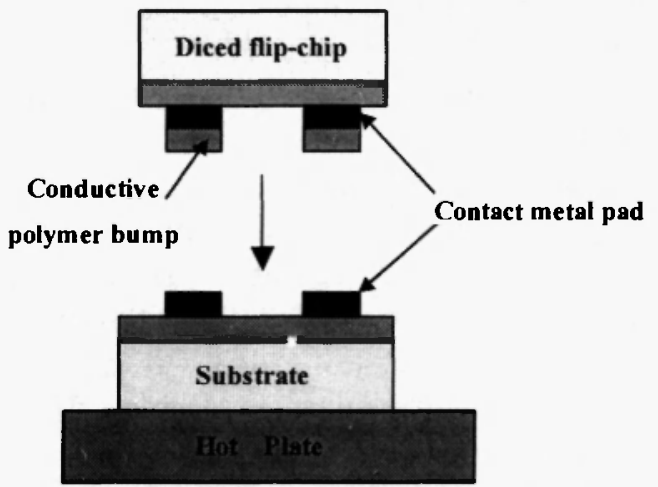

Fig.3 Flip chip bonding technique using micromachined conductive polymer bumps

Fig.3 shows the bonding process of the diced flip chip to the substrate.

The conductive polymer flip chip bonding technique shows a very low constant resistance and offers simple processing steps, a high bumping alignment resolution and a lower bonding temperature. This new bonding technique has a high potential to replace conventional flip chip bonding techniques for sensor and actuator systems, bio/chemical micro total analysis systems ( $\mu$-TAS), optical MEMS, etc.

\subsection{Flip Chip Assembly with silicon removal}

Currently, many MEMS-based RF devices are developed for various microwave and millimeter-wave applications. However, most RF MEMS have to be fabricated using GaAs, ceramics, high resistivity silicon or other RFcompatible substrates. Such fabrication techniques are not commonly used by the mainstream silicon-based MEMS manufacturing infrastructure. As a result, the complexities of these MEMS devices are very limited. Researchers developed flip chip assembly and silicon removal technique [6]. This technique is easily applicable with most standard foundry processes and has been successfully used in the packaging of some RF MEMS devices.

Fig.4 show the assembly and silicon removal processes. Starting with an unreleased MEMS device, indium (or other alloy) is
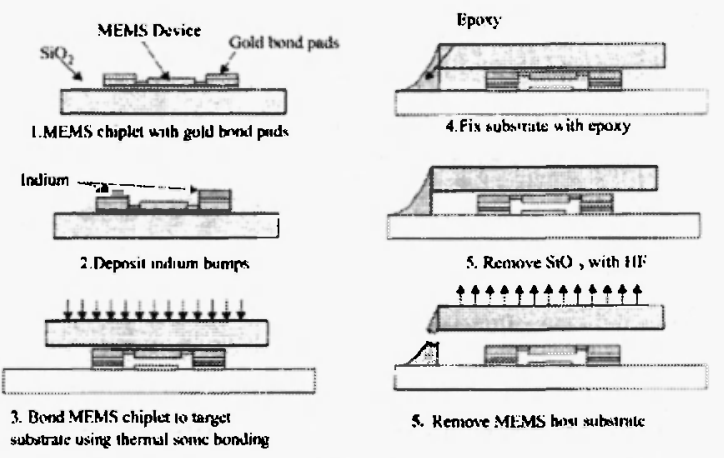

Fig.4 Flip chip assembly and silicon removal deposited onto bonding pads to a desired thickness. The entire structure is then thermosonically flip-chip bonded to a target substrate. With the device attached, the silicon substrate with a deposited epoxy or a sacrificial indium bump. The anchor not only prevents the substrate from contacting the device after release, but the suspended substrate also acts as a shield throughout the release and drying process. Once released, the substrate can be removed safely without damage to the device.

Harsh et al. used this technology to transfer a MEMS tunable capacitor [7]. It is reported the successful device transfer rate is $100 \%$. Experiments show that the assembled devices demonstrate good RF performance, survivability, and packaging control. It is also noticeable that although the flip-chip assembly with silicon removal is easily applicable with most standard foundry processes, some design considerations are necessary creating a device to be flip-chip transferred. Design issues, such as such as warpage, actuator design, temperature distributions, heat sink displacement and structural rigidity, are all crucial considerations when a device is designed to be transferred.

\section{Conclusions}

1. Although MEMS packaging is much more complicated than that for microelectronics, flip chip technologies can be applicable means of integrating MEMS devices.

2. To meet different packaging requirements of 
various MEMS devices, bumping materials, bumping process and joining methods must be chosen carefully.

3. Issues, such as package deformation, temperature distributions, heat sink placement, are all very crucial for the performance of MEMS devices and should be considered in MEMS packaging design.

\section{Acknowledgments}

This work is supported by Chinese Academy of Science.(KJCX2-SW-L2).

\section{References}

[1] Mehalso R., MEMS packaging and Microassembly Challenges, Proceedings of SPIE Vol.3893, Design, Characterization and Packaging for MEMS and Microelectronics, 1999, Queensland, Australia, 22-25

[2] N. P. Kim et al., Aircraft Flight tests and Reliability Improvements of MEMS Pressure
Assembly, Journal of SMT, January 2001, pp. 16.

[3]Jin Y., Zhang J., et al, A novel vacuum packaging for micromachined gyroscope by low temperature solder sealing, Proceedings of the 2001 International Symposium on Electronic Packaging Technology, 270-273

[4] Lau J.,Flip Chip Technologies, McGraw-Hill, NY, 1995

[5] Kwang W., Chong H., A new flip-chip bonding technique using micromachined conductive polymer bumps, IEEE Trans. Advanced Packaging, 22(4) (1999)586-591 [6]Singh, D.S., Horsley, M.B., et al., Batch transfer of microstrurctures using flip-chip solder bump bonding, Transducers 97: 1997 International Conference on Solid-State Sensors and Actuators, Chicago, June 16-19, 1997

[7]. Harsh K.F., Su B.,et al, The realization and design considerations of a flip-chip integrated MEMS tunable capaitor, Sensors and Actuators A Physical, 80(1) (2000), 108-118 